\title{
Heart phantom with electrical properties of heart muscle tissue
}

Leonie Korn, RWTH Aachen University (Chair for Medical Information Technology) Aachen, Germany, korn@hia.rwth-aachen.de

Simon Lyra, RWTH Aachen University (Chair for Medical Information Technology) Aachen, Germany, simon.lyra@rwth-aachen.de

Daniel Rüschen, RWTH Aachen University (Chair for Medical Information Technology) Aachen, Germany, rueschen@hia.rwth-aachen.de

Alexander Pugovkin, National Research University of Electronic Technology (Institute of Biomedical Systems) Moscow, Russia, Pugovkin@bms.zone

Dmitry Telyshev, Sechenov First MSMU (Institute for Bionic Technologies and Engineering) Moscow, Russia, telyshev@,zitc-mt.ru

Steffen Leonhardt, RWTH Aachen University (Chair for Medical Information Technology ) Aachen, Germany, leonhardt@hia.rwth-aachen.de

Marian Walter, RWTH Aachen University (Chair for Medical Information Technology ) Aachen, Germany, walter@hia.rwth-aachen.de

The weakened heart is supported by a left ventricular assist device (LVAD) to supply the heart muscle with oxygenated blood. In case the heart muscle recovers during LVAD therapy, the patient has to be weaned from the device. To date, there is no adequate method to detect heart muscle recovery in LVAD therapy. In order to establish a novel method based on the measurement of electric conductivity, this study presents a silicone model of a ventricle mock-up to simulate the electrical properties of cardiac muscle tissue. Previously, it has been shown that the electrical properties of myocardial tissue change during ischemia, so that these changes are a possible estimate for measuring the condition of myocardial tissue. To this purpose, this study presents a casting process for a ventricle model and describes the materials used to imitate the electrical properties of the heart muscle to obtain conductive material. Initial results showed that the higher the carbon concentration in the silicone, the higher the conductivity of the silicone samples. The measurements were performed at different frequencies and the samples were analyzed for homogenization. 


\section{Regional analysis of airway abnormalities in cystic fibrosis employing Electrical Impedance Tomography}

Sabine Krueger-Ziolek, Institute of Technical Medicine, Furtwangen University, Villingen-Schwenningen, Germany, krue@hs-furtwangen.de

Bo Gong, Institute of Technical Medicine, Furtwangen University, Villingen-Schwenningen, Germany, gon@hsfurtwangen.de

Hanna Zimmermann, Department of Clinical Radiology, LMU Hospital of the University of Munich - Inner City Campus, München, Germany, Hanna.Zimmermann@med.uni-muenchen.de

Ullrich Müller-Lisse, Department of Clinical Radiology, LMU Hospital of the University of Munich - Inner City Campus, München, Germany, ullrich.mueller-lisse@med.uni-muenchen.de

Knut Möller, Institute of Technical Medicine, Furtwangen University, Villingen-Schwenningen, Germany, moe@hsfurtwangen.de

To estimate the severity of airway abnormalities in cystic fibrosis (CF) Brody et al. developed a computed tomography (CT) scoring system. Each pulmonary lobe is analyzed separately considering various morphological defects. A study from Zhao et al. demonstrates that this CT-based score correlates with regional airway obstruction (RAO) measured by the real-time imaging method Electrical Impedance Tomography (EIT). Zhao et al. performed EIT measurements at the $5^{\text {th }}$ intercostal space (ICS) and median RAO, including both lungs, was correlated with the associated score.

In the present feasibility study, it was investigated if RAO determined by EIT within the left and right lung respectively at the $3^{\text {rd }}$ and $5^{\text {th }}$ ICS corresponds with the scores of the left and right lobes. EIT measurements and CT-based scoring were carried out on two CF patients. RAO was identified by ratios of impedance values associated to the maximal forced expiratory flow at $25 \%$ and $75 \%$ of the forced vital capacity. Mean RAO of each lung within both thorax sections was compared with the lobar scores. Airway abnormalities within upper lobes are assigned to RAO measured within the $3^{\text {rd }}$ ICS, whereas abnormalities of the right middle lobe, both lower lobes and the lingula are mainly represented by EIT images of the $5^{\text {th }}$ ICS. Results show that differences in the CT-based score between the left and right lung concur with differences in EIT derived RAO. The regional information provided by EIT might be used for a more targeted therapy of CF-related lung diseases. 


\section{An efficient classification-reconstruction method for 3D EIT}

Bo Gong, Institute of Technical Medicine, VS-Schwenningen, Germany, Bo.Gong@hs-furtwangen.de Sabine Krueger-Ziolek, Institute of Technical Medicine, VS-Schwenningen, Germany, Sabine.Krueger-Ziolek@hsfurtwangen.de

Knut Moeller, Institute of Technical Medicine, VS-Schwenningen, Germany, Knut.Moeller@hs-furtwangen.de

Electrical Impedance Tomography (EIT) is an imaging method which attempts to reveal the conductivity distribution within human body based on the electrical boundary information collected from the electrodes attached on the skin. EIT reconstruction is an ill-posed inverse problem. It is commonly solved within the finite element model framework (FEM). One issue that induces such ill-posedness is the huge number of free parameters associated with the FEM modelling in comparison to the limited number of independent voltage measurements. As a consequence, the optimal solution of the inverse problem is not unique and unstable to noise. To circumvent this problem, different regularization methods have been applied to restrict the freedom the underlying parameters. An attempt to regularize the reconstruction is based on FEM elements clustering, by forcing the FEM elements within each element cluster sharing the same impedance. Hence, the degree of freedom of the inverse problem has been reduced.

In previous studies, the clustering methods either require a patient-specific CT image or have a high computational complexity. In this study, we present an efficient approach of such cluster based regularization by applying a conditional random field (CRF) model. CRF has been applied on an initial reconstruction obtained by applying a fast GaussNewton linear solver. FEM elements with similar conductivity values and geometrical proximities have been grouped in the same cluster. A conjugate gradient solver has been applied on these clusters for fast reconstruction. Employing a 3D FEM mesh with 27,578 nodes, the computation time of the proposed framework is around 2s with an i5-7200U CPU. Initial results indicated that the geometry of the simulated contrasts can be better presented in the reconstructions applying the proposed framework. 


\section{Short Distance Impedance Pneumography}

Michael Klum, Chair of Electronics and Medical Signal Processing, Technische Universität Berlin, Berlin, Germany, michael.klum@tu-berlin.de

Tianhao Schenck, Alexandru Pielmus, Timo Tigges, Reinhold Orglmeister Chair of Electronics and Medical Signal Processing, Technische Universität Berlin, Berlin, Germany, michael.klum@tu-berlin.de

Gold-standards for biosignal acquisition require body-spanning sensor positioning which is contradictory to the high integration of modern wearable medical monitors. In applications where obtrusiveness can decrease accuracy, as in sleep monitoring, compact sensor configurations are not only a matter of convenience. To acquire respiratory signals, most systems rely on nasal cannula pressure sensors or inductance plethysmography. Another well-established method is the impedance pneumography, where we aim to contribute to the field of short distance electrode configurations. Evaluating distances down to $8 \mathrm{~cm}$ we report linear correlations above 0.85 with respect to a pneumotachometer reference. We estimate the respiratory rate with an error below $0.2 \mathrm{bpm}$. Inspiratory and expiratory phase detection is possible with an error below $2.5 \%$. Using a first order polynomial model we estimated the respiratory flow with a relative error of down to $19 \%$ at $8 \mathrm{~cm}$. We conclude that short distance impedance pneumography is feasible and rough flow and volume estimates are possible using linear models. Further research regarding shorter distances and calibration is of great interest. 


\section{Feature based analysis of the cardiosynchronous EIT signal component using an MRI-based reconstruction model}

Michael Kircher, Institute of Biomedical Engineering, Karlsruhe Institute of Technology, Karlsruhe, Germany, michael.kircher@kit.edu

Robert Menges, Institute of Biomedical Engineering, Karlsruhe Institute of Technology, Karlsruhe

Olaf Dössel, Institute of Biomedical Engineering, Karlsruhe Institute of Technology, Karlsruhe

Mechanical ventilation could potentially be optimized clinically if regional pulmonary blood perfusion could be monitored at bedside. The cardiosynchronous component of an EIT measurement has been suggested as potential surrogate for pulmonary blood perfusion, though the origin of this cardiac pulsatility must be understood fully. To contribute to its understanding, a small pilot study with two subjects has been conducted. The subjects were asked to breath at respiration rates of 8,15 and 24 breaths/min as well as spontaneously for $1 \mathrm{~min}$ each in supine position. Additionally, the subjects were asked to hold their breath at inspiration and expiration for 40s. EIT and 3-lead ECG measurements were conducted synchronously and continuously. EIT measurements were carried out using the PV500 at 50 frames/s. The electrode positions were marked with MRI markers and an MRI scan gated at inspiration was conducted the same day. The thorax contour and the electrode positions were segmented from the MRI volume and a subject specific reconstruction model with a homogenous background conductivity was created. The EIT images were reconstructed using a 1-step Gauss-Newton solver. The cardiac pulsatility was extracted by ECG gating and averaging was performed over ten consecutive RR intervals. Three features were extracted from the averaged pulsatility curves: the peak to peak amplitude and the steepest positive and negative temporal slope. The feature distribution images were analysed and compared between the experimental steps. The steepest positive slope distribution map represented mainly heart or ventricular pixel, while the steepest negative slope mainly represented lung regions within the EIT image space. This finding might be an indicator for the theory, that cardiac pulsatility in humans and this specific setup represents regional blood volume change, since the regional impedance should increase rapidly in heart pixels during the systolic contraction, while the impedance within lung regions might decrease quickly. 


\section{"Bioimpedance Analysis of L929 and HaCaT Cells in Low Frequency Range"}

Viviane S. Teixeira, Institute for Medical and Nanoelectronics, Hamburg University of Technology, Eissendorfer Str. 38, 21073 Hamburg, Germany, viviane.silva.teixeira@tuhh.de

Jan-Patrick Kalckhoff, Institute for Medical and Nanoelectronics, Hamburg University of Technology, Eissendorfer Str. 38, 21073 Hamburg, Germany, jan.kalckhoff@tuhh.de

Wolfgang Krautschneider, Institute for Medical and Nanoelectronics, Hamburg University of Technology, Eissendorfer Str. 38, 21073 Hamburg, Germany, krautschneider@tuhh.de

Dietmar Schroeder, Institute for Medical and Nanoelectronics, Hamburg University of Technology, Eissendorfer Str.

38, 21073 Hamburg, Germany, d-schroeder@tuhh.de

In this work, Bioimpedance Spectroscopy (BIS) is used to study fluids and cell solutions. A new four-electrode-terminal (4T) chamber using 3D printing and stainless steel corrosion resistant (V4A) was designed to measure the impedance of live cell solutions at the frequency range $0.1 \mathrm{~Hz}-1 \mathrm{MHz}$. At frequencies below $1 \mathrm{kHz}$ the double layer (DL) that builds at electrode's surface raises the impedance substantially preventing the observation of the real impedance of the cells. The new $4 \mathrm{~T}$ design circumvents the DL, is more robust and cheap, and allows for the repeatability of the results. Experiments were performed in vitro with two cell lines, L929 (mouse fibroblasts) and HaCaT (human keratinocytes). Results show that it is possible to distinguish between the two cell lines by means of its BIS measurements in the new setup. Also, a low-frequency dispersion ( $\alpha$-dispersion) was observed in HaCaT cells solution, but not in L929. By the end, a potentiostat circuit model was developed in LTSpice to simulate the hardware setup and two different circuit models were used to fit cell's data. 